count and/or ultrasounds) were enrolled in this 5-years prospective observational study. Clinical assessments as well as joint ultrasound (US) and MRI were performed at baseline and after 5 years. Each patient underwent a non-dominant hand-wrist US examination using a Logiq 9 with a linear probe operating at 14 $\mathrm{MHz}$. Synovitis was defined as the presence of synovial hypertrophy and/or the presence of power Doppler signal (PD). A non-dominant hand-wrist MRI study with a $0.3 \mathrm{~T}$ extremity dedicated machine to evaluate the presence of bone erosions (BE) and bone marrow edema (BME) was also performed. Coronal and axial T1-weighted gradient-echo images and coronal STIR images were acquired. The images acquired were scored according to the RAMRIS scoring system for RA by a trained radiologist unaware of the clinical picture and diagnosis.

Results: Fifty-seven pts were enrolled (female $91.2 \%$, mean age $44 \pm 12.2$ years, mean disease duration $15.9 \pm 9$ years); 43 (75.4\%) completed the follow-up, 3 died $(5.2 \%)$ and $11(19.4 \%)$ were lost to follow-up. At baseline, 7 (12.3\%)satisfied criteria for Jaccoud arthropathy $(\mathrm{JA})$ and $7(12.3 \%)$ had a recent onset arthritis ( $<1$ year of duration). 22 pts (28.6\%) showed clinical signs of synovitis, 56 (98.2\%) presented positive hand-wrists US (synovitis) and in 14 (24.6\%) PD signal was also recorded. Six pts $(11.76 \%)$ already showed erosions at standard X-Ray, while $M R I$ revealed at least one $B E$ in 30 and 54 patients respectively, for a cumulative mean erosive burden of 9.2 erosions (range 1-63). After 5 years of follow-up, 34 pts consented to repeat the assessment; $11(33 \%)$ had JA and 18 (29\%) were still presenting clinical signs of synovitis; 28 pts $(82.3 \%)$ showed synovitis at US with PD in 7 cases $(20.5 \%)$. The final mean erosive burden resulted 12.3 (range 2-82) with a significant increase from the baseline evaluation $(p=0.001)$. Overall, 16 pts accrued joint damage. Interestingly, erosion progression was observed also in 12 pts with negative joint count but positive US at baseline. The presence of PD at US and BME at baseline was associated with higher erosive burden at follow-up ( $p=0.03$ and $p=0.02$ respectively)

Conclusions: Arthritis in SLE can be persistent over time and progress to joint damage even in a short tem period despite treatment; normal joint count at physical examination but US findings of synovitis can be associated with erosion progression over time. The presence of PD at US and bone marrow edema at $\mathrm{MRI}$ are associated with a more severe damage progression. US and MRI can be a valid help for the clinician to identify patients at higher risk of severe damage to be treated with a more aggressive therapeutic strategy targeting arthritis.

Disclosure of Interest: None declared

DOI: 10.1136/annrheumdis-2017-eular.6016

\section{FRI0641 WRIST INFLAMMATION AS ASSESSED BY MAGNETIC RESONANCE IMAGING IS ASSOCIATED WITH PATIENT- REPORTED PHYSICAL IMPAIRMENT, GLOBAL DISEASE ACTIVITY AND PAIN IN EARLY RHEUMATOID ARTHRITIS: LONG-TERM RESULTS FROM TWO RANDOMIZED CONTROLLED TRIALS}

D. Glinatsi ${ }^{1}$, J.F. Baker ${ }^{2}$, M.L. Hetland ${ }^{1,3}$, K. Hørslev-Petersen ${ }^{4,5}$, B.J. Ejbjerg ${ }^{6}$, K. Stengaard-Pedersen ${ }^{7}$, P. Junker ${ }^{8}$, T. Ellingsen ${ }^{8}$, H.M. Lindegaard ${ }^{8}$, I. Hansen ${ }^{7}$, T. Lottenburger ${ }^{9}$, J.M. Møller ${ }^{10}$, L. Ørnbjerg ${ }^{1}$, A. Vestergaard ${ }^{11}$ A.G. Jurik ${ }^{7,12}$, H.S. Thomsen ${ }^{3,10}$, T. Torfing ${ }^{8}$, S.M. Bisgaard ${ }^{1}$, M.B. Axelsen ${ }^{1}$, M. Østergaard ${ }^{1,3} \cdot{ }^{1}$ Rigshospitalet, Glostrup, Denmark; ${ }^{2}$ Corporal Michael J. Crescenz VA Medical Center, Philadelphia, PA, United States; ${ }^{3}$ University of Copenhagen, Copenhagen; ${ }^{4}$ King Christian 10th Hospital for Rheumatic Diseases, Gråsten: ${ }^{5}$ University of Southern Denmark, Odense: ${ }^{6}$ Slagelse Hospital, Slagelse: ${ }^{7}$ Aarhus University Hospital, Aarhus; ${ }^{8}$ Odense University Hospital, Odense; ${ }^{9}$ Vejle Hospital, SLB, Vejle; ${ }^{10}$ Copenhagen University Hospital Herlev, Herlev; ${ }^{11}$ Hvidovre University Hospital, Hvidovre; ${ }^{12}$ Aarhus University, Aarhus, Denmark

Background: Studies in established rheumatoid arthritis (RA) have shown that radiographic progression is associated with increasing health assessment questionnaire (HAQ) score. However, most studies have failed to demonstrate this association at the early stage of the disease. In addition, little is known about how specific pathologies, e.g. joint inflammation, tenosynovitis and joint damage, contribute to different patient-reported outcomes (PROs).

Objectives: To examine the association between MRI wrist inflammation and damage with PROs in patients with early RA.

Methods: MRIs of the wrist and hand were obtained from 210 early RA patients participating in two investigator-initiated, randomized, controlled studies (CIMESTRA/OPERA), which aimed at achieving inflammatory control by use of conventional and/or biologic drugs combined with intra-articular injection of glucocorticoids. The image-sets were assessed according to the RA MRI scoring system (RAMRIS) for inflammation (synovitis, tenosynovitis, osteitis) and damage (bone erosions, joint space narrowing) at baseline $(n=210), 1 \quad(n=206)$ and 5 $(n=113)$ years follow-up. Data from the two studies were pooled and assessed for associations between MRI features and $\mathrm{HAQ}$, patient global visual analogue scales (VAS-PtGlobal) and VAS pain using Spearman correlation for status and change scores, univariate and multivariable linear regression analyses for change scores and generalized estimating equations for status and change scores. MRI features were further tested for trends against specific hand-related HAQ questions using the Jonckheere trend test.

Results: MRI inflammation, but not damage, showed statistically significant associations with HAQ, VAS-PtGlobal and VAS pain for status and change scores, independently of swollen joint count and level of C-reactive protein.
Synovitis and tenosynovitis were the MRI features most consistently associated with PROs, particularly VAS-PtGlobal and VAS pain (Table 1). MRI synovitis and tenosynovitis mean scores increased with the level of difficulty to cut meat and open a milk carton $(p<0.01)$, and similar patterns were seen for other hand-related $\mathrm{HAQ}$ items. As an additional analysis, the metacarpophalangeal joints were included in the analyses, but this did not strengthen the associations between MRI pathology and PROs.

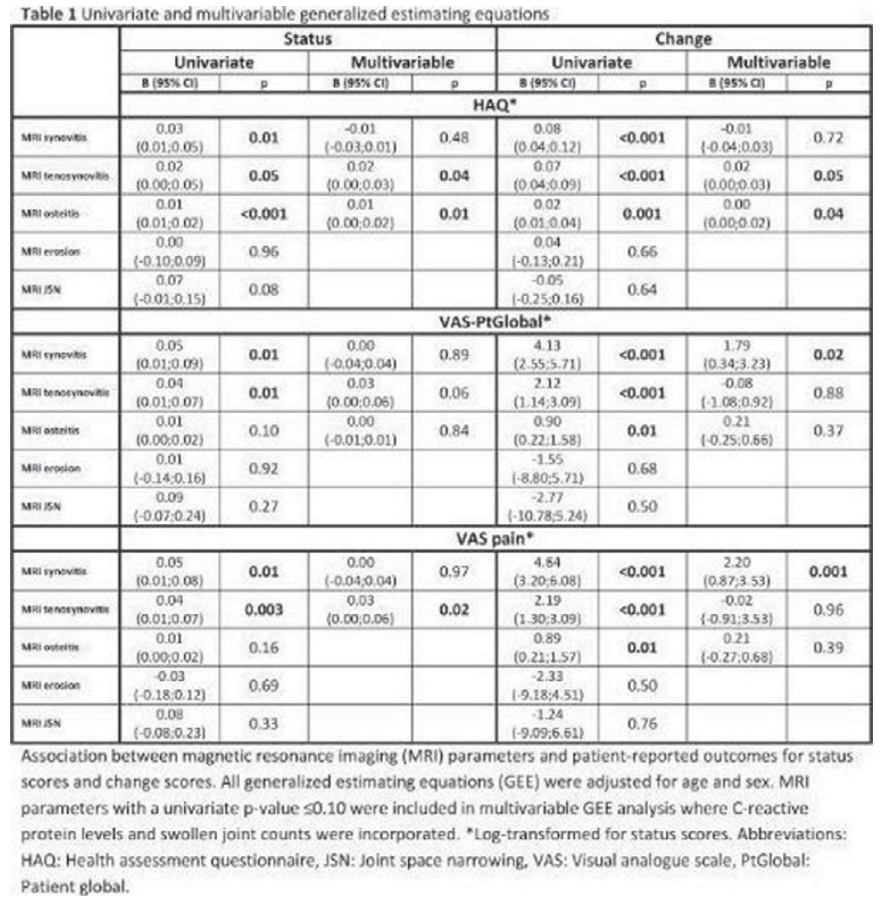

Conclusions: MRI-assessed inflammation, but not damage, in the early RA wrist is associated with patient-reported physical impairment, global disease activity and pain, and the amount of wrist inflammation influences physical hand function. Disclosure of Interest: D. Glinatsi: None declared, J. Baker: None declared, M. Hetland Grant/research support from: BMS, AbbVie, Pfizer, UCB-Nordic, MSD, K. Hørslev-Petersen Consultant for: AbbVie and UCB, B. Ejbjerg: None declared, K. Stengaard-Pedersen: None declared, P. Junker: None declared, T. Ellingsen: None declared, H. Lindegaard: None declared, I. Hansen: None declared, T. Lottenburger: None declared, J. Møller: None declared, L. Ørnbjerg: None declared, A. Vestergaard: None declared, A. G. Jurik: None declared, H. Thomsen: None declared, T. Torfing: None declared, S. Bisgaard: None declared, M. Axelsen Grant/research support from: Abbvie, M. Østergaard Grant/research support from: Abbvie, BMS, Janssen and Merck, Consultant for: Abbvie, BMS, Boehringer-Ingelheim, Celgene, Eli-Lilly, Centocor, GSK, Hospira, Janssen, Merck, Mundipharma, Novartis, Novo, Orion, Pfizer, Regeneron, Schering-Plough, Roche, Takeda, UCB, and Wyeth

DOI: 10.1136/annrheumdis-2017-eular.1555

\section{FRI0642 THE POTENTIAL VALUE OF POSITRON EMISSION TOMOGRAPHY (PET)-SCAN IN SYSTEMIC SCLEROSIS FOR THE QUANTITATIVE ASSESSMENT OF INTERSTITIAL LUNG DISEASE}

D.M. Peelen ${ }^{1}$, B. Zwezerijnen ${ }^{2}$, E.J. Nossent ${ }^{3}$, L.J. Meijboom ${ }^{4}$, O.S. Hoekstra ${ }^{2}$, C.J. van der Laken ${ }^{1}$, A.E. Voskuyl ${ }^{1} .{ }^{1}$ Rheumatology; ${ }^{2}$ Nuclear Medicine; ${ }^{3}$ Pulmonology: ${ }^{4}$ Radiology, VU University Medical Center, Amsterdam, Netherlands

Background: Interstitial lung disease (ILD) in systemic sclerosis is treated by immunosuppressive drugs (e.g. cyclophosphamide), aimed at reduction of inflammatory response. Differentiation between inflamed and non-inflamed fibrotic tissue might help to develop treatment stratification, with the aim of improving the prognosis of (subgroups of) SSc-ILD patients. ${ }^{18}$ F-Fluoro-Dexoxyglucose Positron Emission Tomography $\left({ }^{18} \mathrm{~F}\right.$-FDG PET) scan might be a promising tool to detect inflamed lung areas, as formerly shown in a semi-quantitative setting.[1,2] Objectives: This study aims to investigate the potential role of ${ }^{18}$ F-FDG PET -scan for the quantitative assessment of metabolically active SSc related ILD.

Methods: ${ }^{18} \mathrm{~F}$-FDG PET -scans of 22 patients with systemic auto-immune disease, including 9 with SSc, 9 with systemic lupus erythematosus (SLE) and 4 with primary Sjögren's syndrome (pSS), were retrospectively analyzed. FDGuptake was quantitatively measured within $2 \mathrm{~cm}$-sized Regions of Interest (ROI's) at apical, medial and basal lung levels. A total of 22 ROl's were drawn in each patient. SUVmean values of all ROI's were corrected by the medial SUVmean bloodpool value. Subsequently, the average of 6 posterior basal SUVmean values 
was divided by the average of 6 posterior apical SUVmean values (basal/apical ratio). High Resolution Computed tomography (HRCT)-scans and Pulmonary Function Tests (PFT) were examined to confirm the diagnosis of ILD and to specify the pattern of fibrosis.

Results: Mean age of patients was 69.4 (SSc-ILD), 62.5 (SSc without ILD), 38.9 (SLE) and, 49.3 (pSS). In SSc patients, the mean disease duration was 5.0 (SSc-ILD) and 4.4 (SSc without ILD) years. Diffuse cutaneous sclerosis was present in 2 SSc-ILD and 1 SSc without ILD patients, while other SSc patients were diagnosed with limited cutaneous SSc. ILD was present in 5 out of 9 SSc patients as confirmed by HRCT and PFT. ILD was active in 3 out of 5 SSc-ILD patients. Posterior basal/apical SUVmean ratios of SSc-ILD patients were significantly increased compared to SSc patients without ILD $(p=0.016)$, and compared to SLE and pSS patients without ILD ( $p=0.001$ and $p=0.016$, respectively), which is shown in Figure 1.

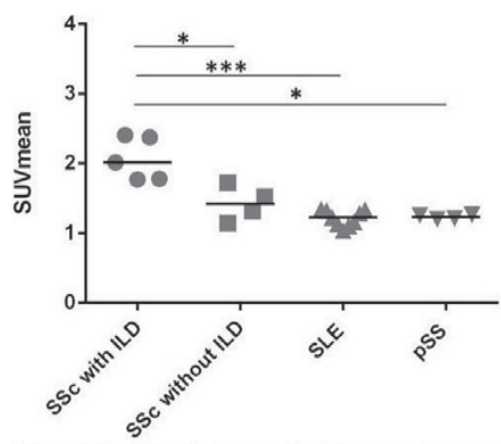

Figure 1: Basal/Apical ratio of SUVmean scores (corrected for bloodpool) in systemic autoimmune patients.

$*=p<0.05,{ }^{* * *}=p<0.001$ (Mann-Whitney test), $-=$ median

Conclusions: Our findings demonstrate that ${ }^{18} \mathrm{~F}-\mathrm{FDG}$ PET -scan is potentially useful for the quantitative assessment of active ILD lesions in SSc patients. The technique may therefore provide opportunities to select the patients with inflammatory regions in ILD that are most likely to respond to immunesuppression. References:

[1] Uehara, T., et al., Deep-inspiration breath-hold 18F-FDG-PET/CT is useful for assessment of connective tissue disease associated interstitial pneumonia. Mod Rheumatol, 2016. 26(1): p. 121-7.

[2] Jacquelin, V., et al., FDG-PET/CT in the prediction of pulmonary function improvement in nonspecific interstitial pneumonia. A Pilot Study. Eur J Radiol, 2016. 85(12): p. 2200-2205

Disclosure of Interest: None declared

DOI: 10.1136/annrheumdis-2017-eular.3677

\section{FRI0643 ENTHESITIS AND FOOT DISABILITY IN PATIENTS WITH ANKYLOSING SPONDYLITIS}

A. Serce, A. Bal, D. Dulgeroglu, A. Cakci. Physical Medicine and Rehabilitation, Diskapi YB Education and Resarch Hospital, Ankara, Turkey

Background: Enthesitis is a primary clinical feature of ankylosing spondylitis (AS). Lower extremity enthesopathy may cause foot pain and functional disability. Detection of enthesitis by using physical examination may be insufficient. Musculoskeletal ultrasonography is a noninvasive and low-cost method for detecting enthesitis readily.

Objectives: The aims of this study were to evaluate enthesitis by using musculoskeletal ultrasonography and foot disability in patients with AS, to compare healthy controls, and to determine the correlation of enthesitis score and foot function with disease activity and functional status.

Methods: In this study, 101 patients with AS and 42 healthy controls were examined for enthesal site abnormalities by using gray-scale ultrasonography. The findings were assessed by using the Glasgow Ultrasound Enthesitis Scoring System (GUESS). The foot function index (FFI), which comprised of pain, disability, and activity limitation subscales, was measured in all the patients with AS and healthy controls for assessment of foot function. Disease activity and functional status were assessed using the Bath AS Disease Activity Index (BASDAl) and Bath AS Functional Index (BASFI), respectively, in patients with AS.

Results: The median GUESS score was 8.00 (1.00-23.00), and, the median total FFI and scores in all the pain, disability, and activity limitation subscales were $14.70(0.00-75.20), 16.60(0.00-82.80), 16.10(0.00-84.40)$, and 4.00 $(0.00-60.00)$, respectively in patients with AS. The GUESS score, total FFI, and all the subscales scores were significantly higher in the patients with AS than in the controls $(p=0.00)$. GUESS score showed no correlation with BASDAI and BASFI. In patients with AS, total FFI and scores for all subscales showed positive correlations between BASDAI and BASFI, respectively $(p=0.00, r=0,66 ; p=0.00$, $r=0,0,50 ; \mathrm{p}=0.00, r=0,59 ; \mathrm{p}=0.00, r=0,31 ; \mathrm{p}=0.00, r=0,60 ; \mathrm{p}=0.00, r=0,54 ; \mathrm{p}=0.00$, $r=0.57 ; \mathrm{p}=0.00, r=0.50)$.

Conclusions: The severities of enthesitis and foot disability were higher in patients with AS. Patients with AS may undergo ultrasonographic examination for enthesal foot involvement. Foot disability is related with disease activity and function. Foot involvement and disability should be evaluated comprehensively and managed properly.

\section{References:}

[1] Balint PV, Kane D, Wilson H, McInnes IB, Sturrock RD. Ultrasonography of enthesal insertions in the lower limb in spondyloarthropathy. Ann Rheum Dis 202;61:905-910.

[2] Borman P, Koparal S, Babaoğlu S, Bodur H. Ultrasound detection of entheseal insertions in the foot of patients with spondyloarthropathy. Clin Rheumatol 2006:25:373-377.

Disclosure of Interest: None declared

DOI: 10.1136/annrheumdis-2017-eular.5112

\section{FRI0644 SUBCLINICAL ULTRASONOGRAPHIC CHANGES OF THE ANTERIOR CHEST WALL JOINTS IN ANKYLOSING SPONDYLITIS AND RHEUMATOID ARTHRITIS AND THEIR ASSOCIATION WITH CHEST EXPANSION}

F.l. Abdelrahman ${ }^{1}$, M.A. Mortada ${ }^{1}$, A.B. Abdul Sattar ${ }^{1}$, A.A. El Sammak ${ }^{2}$. ${ }^{1}$ Rheumatology and Rehabilitation; ${ }^{2}$ Radiology, Zagazig University, Zagazig, Egypt

Background: Anterior chest wall (ACW) joints can be involved during the course of Rheumatoid arthritis (RA) and ankylosing spondylitis (AS), however, its clinical implications appear to be underestimated by the rheumatology community. Objectives: To determine the prevelance and types of subclinical ultrasonographic changes in the ACW joints in RA and AS patients and their association with the chest expansion.

Methods: The study was conducted on 132 sternoclavicular joints (SCJ) and 66 manibrusternal joints (MSJ) in 66 subjects (22 AS, 22 RA, and 22 control). Ultrasound (US) assessments were performed to detect synovitis, erosions, ankylosis, osteophytes, or doppler signals. Chest expansion was measured. In RA group, Disease Activity Score (DAS28) and Health Assesment Questionare Disability Index (HAQDI) were recorded. In AS group, Ankylosing Spondylitis Disease Activity Score (ASDAS), Bath Ankylosing Spondylitis Disease Activity Index (BASDAI) and Bath Ankylosing Spondylitis Functional Index (BASFI) were recorded.

Results: US detected subclinical changes of ACW joints in (74.2\%) of RA, (77.3\%) of AS, and $(21.2 \%)$ of control groups. There was a highly significant difference between total US changes in RA $(74.2 \%)$ and control $(21.2 \%)(p<0.001)$ and also between AS $(77.3 \%)$ and control $(21.2 \%)(p<0.001)$. Non of our control had neither erosions nor ankylosing in MSJ. MSJ ankylosing was signficantly higher in AS $(77.3 \%)$ than RA $(18.2 \%)(p<0.001)$. MSJ ankylosing was highly associated with limited chest expansion in both RA and AS $(P<0.001)$. All patients $(100 \%)$ in both groups (RA and AS) with MSJ ankylosing by US had limited chest expansion. In RA group, ultrasonographic changes were found to be higher with smoking, longer disease duration and high DAS28. In AS group, ultrasonographic changes were found to be higher with older age, male sex, smoking, longer disease duration and high BASDAI and BASFI.

Table 1. Comparison between RA and AS as regard ultrasonographic changes of anterior chest wall joints

\begin{tabular}{lcccc}
\hline & RA n (\%) & AS n (\%) & $\chi^{2}$ & p value \\
\hline SCJ synovitis & $34(77.3)$ & $24(54.5)$ & 5 & $\mathbf{0 . 0 2}^{\star}$ \\
SCJ PD activity & $14(31.8)$ & $12(27.3)$ & 0.2 & 0.6 \\
SCJ erosion & $32(72.3)$ & $28(63.6)$ & 0.8 & 0.4 \\
SCJ osteophyte & $4(4.5)$ & $6(13.6)$ & 0.4 & 0.5 \\
MSJ ankylosing & $4(18.2)$ & $14(63.6)$ & 9.2 & $\mathbf{0 . 0 0 2}^{\star}$ \\
MSJ erosion & $9(40.9)$ & $4(18.2)$ & 2.7 & 0.09 \\
MSJ osteophyte & $1(0.5)$ & $1(0.5)$ & 0 & 1 \\
\hline
\end{tabular}

${ }^{*} \mathrm{n}$ : number; $\chi^{2}$ : chi-square test; PD: Power Doppler signals.

Conclusions: US detected subclinical changes of ACW joints in a high percentage of RA and AS patients. No erosions or ankylosing in MSJ were Found in the healthy individuals. MSJ ankylosing is more in AS than RA. Relatively, ankylosing of MSJ by US is highly associated with limited chest expansion in RA and AS. Up to the best of our knowledge, our study was the first study that detected subclinical changes of ACW joints in RA and AS by US.

References:

[1] Rodríguez-Henríquez P, Solano C, Peña A, León-Hernández S,HernándezDíaz C, Gutiérrez $\mathrm{M}$, et al. Sternoclavicular jointinvolvement in rheumatoid arthritis: clinical and ultrasoundfindings of a neglected joint. Arthritis Care Res 2013;65:1177-82

[2] Frank Verhoeven, Xavier Guillot, Marie Godfrin-Valnet, Clément Prati, et al: Ultrasonographic Evaluation of the Anterior Chest Wall in Spondyloarthritis: A Prospective and Controlled Study; J Rheumatol 2015;42;87-92.

Disclosure of Interest: None declared

DOI: 10.1136/annrheumdis-2017-eular.2245 